\title{
Searching for a direct preparation of dihydropyrimidine-5- carboxamides under Biginelli reaction conditions
}

\author{
Irantzu Couto, Imanol Tellitu,* and Esther Domínguez \\ Departamento de Química Orgánica II, Facultad de Ciencia y Tecnología, \\ Universidad del País Vasco - Euskal Herriko Unibertsitatea, 48940 Leioa (Bizkaia), Spain \\ E-mail:imanol.tellitu@ehu.es
}

Dedicated to the memory of our colleague and friend Prof. Rafael Suau

DOI: http://dx.doi.org/10.3998/ark.5550190.0012.209

\begin{abstract}
In this paper it is established a protocol leading to a series of dihydropyrimidine-5-carboxamides under Biginelli reaction conditions starting directly from 3-ketoamides as the 1,3-dicarbonyl component of the process. The best results have been found when chloroacetic acid is used as the catalyst under solvent free conditions.
\end{abstract}

Keywords: Multicomponent, Biginelli, dihydropyrimidines, heterocycles

\section{Introduction}

Organic chemists have found in the development of known and new multicomponent reactions (MCRs) an inspiration to quickly design straightforward entrances to large families of novel compounds, especially when these syntheses are coupled with combinatorial strategies. ${ }^{1}$ Among them, the venerable 3-MCR Biginelli reaction has recently attracted a renewed interest based, primarily, on the discovery of many different catalysts that allow the preparation of the resultant dihydropyrimidines (DHPMs) with excellent results, as opposed to the limited success encountered in the original reports. ${ }^{2}$ In addition, the finding of therapeutic and pharmacological properties $^{3}$ as channel blockers, antihypertensive agents, $\alpha_{1 \text { a }}$ antagonists and neuropeptide $Y$ (NPY) antagonists, for example, ${ }^{4}$ explains the widespread presence of studies related to the "Biginelli compounds" in the specialized literature, ${ }^{5}$ not to mention the fact that several marine alkaloids, with interesting biological activities as well, include the DHPM motif in their structures. ${ }^{6}$ Apart from subtle modifications from the original design, ${ }^{7}$ the Biginelli reaction takes place by mixing an aldehyde (mainly aromatic aldehydes), substituted or unsubstituted urea 
(or thiourea), and an active 1,3-dicarbonyl compound under appropriate conditions, ${ }^{8}$ in combination with a selected catalyst (see Figure 1).<smiles>[Z]C(=O)CC(C)=O</smiles>

\section{Figure 1}

Most of the recent progress of this transformation is revealed from the large amount of publications showing the benefits of a number of different species that are able to catalyze the construction of the Biginelli adducts, ${ }^{9}$ a research that, in the vast majority of cases, has been developed using a 3-ketoester ( $\mathrm{Z}=\mathrm{OR}$ in Figure 1$)$ as the active methylenic component of the reaction. In addition, post-transformations of the DHPM skeleton can be considered as an active field of research that has led to the preparation of a number of heterocycles of great interest. In particular, some of these structural alterations rely on the hydrolysis of the ester group followed by an amidation process to render 5-carboxamide substituted DHPMs ( $Z=$ NHR in Figure 1). ${ }^{10}$ On the other hand, it has to be mentioned that several examples of such type of derivatives have attracted the attention of medicinal chemists due to their well-recognized properties, examples of which are shown in Figure 2. Of special interest are the N3-acylated DHPMs, such as SNAP 6201 1, which are known to be excellent calcium channel modulator, as well as selective $\alpha_{1 \mathrm{a}}$ adrenergic receptor antagonists. ${ }^{3,5 b}$ Similarly, DHPMs of type 2 have also shown good binding affinity $(<1 \mathrm{nM})$ and excellent subtype selectivity for the $\alpha_{1 \mathrm{a}}$ adrenergic receptor. ${ }^{11}$ In addition to the mitotic kinesin Eg5 inhibitor monastrol, screening of a library of related compounds in phenotype-based screens also led to the DHPM 3 which showed the colchicine-like property of destabilizing microtubules. ${ }^{12}$ Finally, the preparation of $\mathbf{4}$, which was found to have Ki values of 2.9, 537, and $1513 \mathrm{nM}$ vs the $\alpha_{1 \mathrm{a}}, \alpha_{1 \mathrm{~b}}$ and $\alpha_{1 \mathrm{~d}}$ receptors, respectively, has been also described. ${ }^{11}$<smiles>[R]NC(=O)N1C(=O)N([TlH])C(CC)=C(C(N)=O)C1c1ccc(F)c(F)c1</smiles><smiles>[R]NC(=O)C1=C(COC)NC(=O)N[C]1c1ccc(F)c(F)c1</smiles><smiles>[R]=[As][As]CCN1CCC(c2ccc(F)cc2)CC1</smiles><smiles>CC1=C(C(=O)Nc2ccccc2)C(c2ccc3c4c(cccc24)CC3)NC(=S)N1</smiles><smiles>[R]NC(=O)C1=C(C)NC(=O)N(C(=O)OC)C1c1ccc([N+](=O)[O-])cc1</smiles><smiles>COC(=O)C1(c2ccccc2)CCN(CCCC#P)CC1</smiles> 


\section{Figure 2}

Our own overview of the related literature has led to the conclusion that, in addition to the comparatively insignificant amount of reports including the construction of DHPM-5carboxamides starting directly from 3-ketoamides, ${ }^{13}$ there is no account, to the best of our knowledge, in which a specific optimization protocol has been established for the one-step preparation of such kind of derivatives. Moreover, the resulting derivatives can be considered interesting starting points for future post-modifications. This paper shows our results in this area.

\section{Results and Discussion}

\section{Selection of the catalyst}

Initially, we explored the action of a series of catalysts with the aim of selecting the best protocol to ensure an efficient synthesis of a number of DHMP-5-carboxamides of type 8. The catalysts under study were selected on the basis that (1) all of them had succeeded when applied to the same reaction employing 3-ketoesters, (2) they must be economically advantageous, (3) strongly acidic catalysts should be avoided to prevent undesired processes in highly functionalized substrates, and (4) the reaction and the work-up have to be as simple as possible. With these conditions, seven catalysts were selected and applied to our model reaction, exemplified in Scheme 1, that includes in all cases commercially available substrates. Thus, the reaction between acetoacetanilide 5a, benzaldehyde 7a and urea 6a (molar rate 1:1:1.5) was set up in the presence of $10 \mathrm{~mol} \%$ of a series of catalysts 1-7 in the indicated solvent (assays 1-4) or under solvent free conditions (assays 5-7) at the particular temperature.
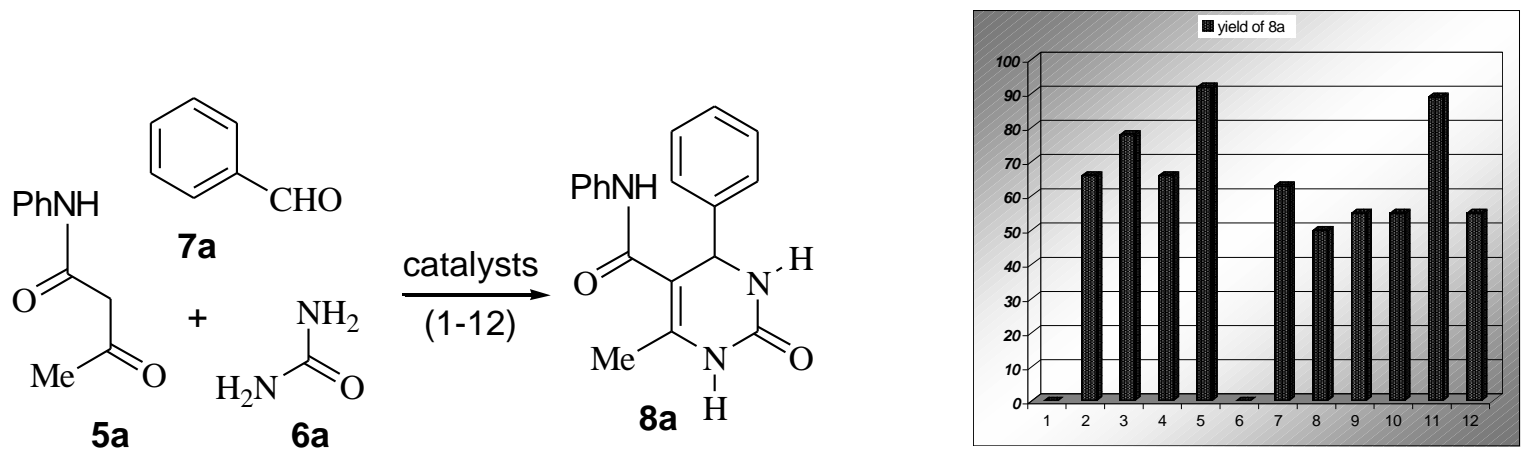

cat. 1: $\mathrm{CaF}_{2}, \mathrm{EtOH}, 40{ }^{\circ} \mathrm{C}, 8 \mathrm{~h}$; cat. 2: $\mathrm{SiCl}_{4}, \mathrm{MeCN} / \mathrm{DMF}(2 / 1)$, rt, $72 \mathrm{~h}$; cat. 3: $\mathrm{SbCl}_{3}, \mathrm{MeCN}$, reflux, $24 \mathrm{~h}$; cat. 4: tartaric acid, $\mathrm{MeOH}$, reflux, $18 \mathrm{~h}$; cat. 5: $\mathrm{ClCH}_{2} \mathrm{COOH}, 90^{\circ} \mathrm{C}, 7 \mathrm{~h}$; cat. 6: L-pro, rt, $18 \mathrm{~h}$; cat. 7: $\mathrm{Ce}\left(\mathrm{NO}_{3}\right) \cdot 6 \mathrm{H}_{2} \mathrm{O}, 80$ ${ }^{\circ} \mathrm{C}$; cat. 8: ${ }^{14} \mathrm{AcOH}$, reflux; cat. 9: ${ }^{8 \mathrm{~b}} \mathrm{AcOH} / \mathrm{EtOH} / \mu \mathrm{w}, 120{ }^{\circ} \mathrm{C}$; cat. 10: ${ }^{8 \mathrm{~b}} \mathrm{AcOH} / \mathrm{EtOH} / \mathrm{Yb}(\mathrm{OTf})_{3}, 120{ }^{\circ} \mathrm{C}$; cat. 11:13c Tungstophosphoric acid, $80{ }^{\circ} \mathrm{C}$; cat. 12: ${ }^{15} \mathrm{AcOH} / \mathrm{EtOH} / \mathrm{CF}_{3} \mathrm{SO}_{3} \mathrm{H} / \mathrm{YbCl}_{3}, 120^{\circ} \mathrm{C}$.

Scheme 1. Experimental (cat 1-7) and reported values (cat 8-12) for DHPM 8a. 
The graphic (see Scheme 1) shows that the expected cyclization takes place in moderate to good yields in five out of the seven assays. The superior conditions ( $92 \%$ yield) were found when chloroacetic acid was used to assist the reaction, and slightly diminished results were obtained when catalysts 2, 3, 4 and 7 were employed. On the contrary, while the use of calcium fluoride resulted in a complex mixture of unidentified compounds, the use of L-proline did not promote the desired cyclization at all, and unreacted substrates were the only materials detected. The benefits from the use of chloroacetic acid over previously reported conditions are highlighted in the last portion of the graphic. Thus, previous reported syntheses of DHPM 8a assisted by the use of catalysts 8-12 have shown diminished results except for catalyst 11 , for which a comparable yield has been reported. However, tungstophosphoric acid $\left(\mathrm{H}_{3} \mathrm{PW}_{12} \mathrm{O}_{40}\right)$ is not commercially available and must be prepared. ${ }^{16}$ In addition, solvent-free reactions have many advantages and important aspects are reduced pollution, lower costs and the simplicity of the processes involved. ${ }^{17}$

\section{Extension to the preparation of a series of DHMP-5-carboxamides (8a-t)}

Once the best conditions have been established, a series of DHPM-5-carboxamides 8a-t were prepared by heating, under solvent free conditions, mixtures of 3-ketoamides 5a-d, substituted or unsubstituted ureas 6a-d, and aromatic or aliphatic aldehydes 7a-i in the presence of $10 \mathrm{~mol} \%$ of chloroacetic acid for the required time (7-10 hours).
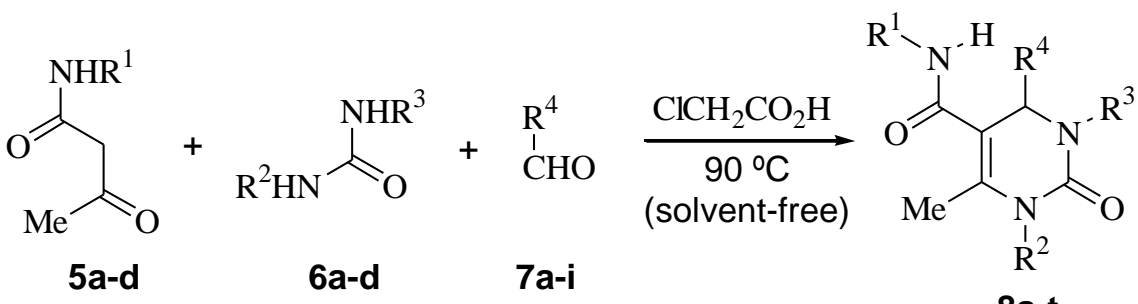

8a-t

Table 1. Synthetic data for the series of DHPMs 8a-t prepared. Yields for isolated compounds

\begin{tabular}{cccccccccc}
\hline $\mathbf{R}^{\mathbf{1}}$ & $\mathbf{R}^{\mathbf{2}}$ & $\mathbf{R}^{\mathbf{3}}$ & $\mathbf{R}^{\mathbf{4}}$ & $\mathbf{8}(\boldsymbol{\%})$ & $\mathbf{R}^{\mathbf{1}}$ & $\mathbf{R}^{\mathbf{2}}$ & $\mathbf{R}^{\mathbf{3}}$ & $\mathbf{R}^{\mathbf{4}}$ & $\mathbf{8}(\boldsymbol{\%})$ \\
\hline $\mathrm{Ph}$ & $\mathrm{H}$ & $\mathrm{H}$ & $\mathrm{Ph}$ & $\mathbf{8 a}(92)$ & $\mathrm{H}$ & $\mathrm{Me}$ & $\mathrm{H}$ & $\mathrm{Ph}$ & $\mathbf{8 k}(73)$ \\
$\mathrm{Ph}$ & $\mathrm{H}$ & $\mathrm{H}$ & $3-\mathrm{MeOC}_{6} \mathrm{H}_{4}$ & $\mathbf{8 b}(63)$ & $\mathrm{Ph}$ & $\mathrm{Me}$ & $\mathrm{H}$ & $3-\mathrm{MeOC}_{6} \mathrm{H}_{4}$ & $\mathbf{8 1}(97)$ \\
$\mathrm{Ph}$ & $\mathrm{H}$ & $\mathrm{H}$ & $2-\operatorname{thienyl}$ & $\mathbf{8 c}(89)$ & $\mathrm{Ph}$ & $\mathrm{Me}$ & $\mathrm{Me}$ & $3-\mathrm{MeOC}_{6} \mathrm{H}_{4}$ & $\mathbf{8 m}(36)$ \\
$\mathrm{Ph}$ & $\mathrm{H}$ & $\mathrm{H}$ & $4-\mathrm{NO}_{2} \mathrm{C}_{6} \mathrm{H}_{4}$ & $\mathbf{8 d}(36)$ & $\mathrm{Ph}$ & $\mathrm{Me}$ & $\mathrm{Me}$ & $\mathrm{Ph}$ & $\mathbf{8 n}(43)$ \\
$\mathrm{Ph}$ & $\mathrm{Me}$ & $\mathrm{H}$ & $\mathrm{Ph}$ & $\mathbf{8 e}(75)$ & $4-\mathrm{NO}_{2} \mathrm{C}_{6} \mathrm{H}_{4}$ & $\mathrm{Me}$ & $\mathrm{Me}$ & $\mathrm{Ph}$ & $\mathbf{8 0}(42)$ \\
$\mathrm{Ph}$ & $\mathrm{Me}$ & $\mathrm{H}$ & $3-\mathrm{HOC}_{6} \mathrm{H}_{4}$ & $\mathbf{8 f}(93)$ & $\mathrm{Ph}$ & $\mathrm{Et}$ & $\mathrm{Et}$ & $3-\mathrm{MeOC}_{6} \mathrm{H}_{4}$ & $\mathbf{8 p}(21)$ \\
$\mathrm{Ph}$ & $\mathrm{Me}$ & $\mathrm{H}$ & $2-\mathrm{BrC}_{6} \mathrm{H}_{4}$ & $\mathbf{8 g}(75)$ & $2,4-\mathrm{Me}_{2} \mathrm{C}_{6} \mathrm{H}_{3}$ & $\mathrm{Me}$ & $\mathrm{Me}$ & $\mathrm{Ph}$ & $\mathbf{8 q}(51)$ \\
$\mathrm{Ph}$ & $\mathrm{Me}$ & $\mathrm{H}$ & ${ }^{\mathrm{Pr}}$ & $\mathbf{8 h}(27)$ & $\mathrm{H}$ & $\mathrm{Me}$ & $\mathrm{Me}$ & $\mathrm{Ph}$ & $\mathbf{8 r}(23)$ \\
$\mathrm{Ph}$ & $\mathrm{Me}$ & $\mathrm{H}$ & ${ }^{\mathrm{Bu}}$ & $\mathbf{8 i}(47)$ & $\mathrm{Ph}$ & $\mathrm{Me}$ & $\mathrm{Me}$ & $2-\mathrm{thienyl}_{1}$ & $\mathbf{8 s}(40)$ \\
$\mathrm{H}$ & $\mathrm{H}$ & $\mathrm{H}$ & $\mathrm{Ph}$ & $\mathbf{8 j}(94)$ & $\mathrm{Ph}$ & $\mathrm{Me}$ & $\mathrm{Me}$ & $2-\mathrm{NO}_{2} \mathrm{C}_{6} \mathrm{H}_{4}$ & $\mathbf{8 t}(42)$ \\
\hline
\end{tabular}


The identity of the new heterocyclic compounds was established by spectroscopic means or by comparison with reported data. A clarifying piece of information comes from the ${ }^{1} \mathrm{H}$ NMR spectrum $\left(\mathrm{CDCl}_{3}\right)$ which reveals a singlet at $\sim 5.5 \mathrm{ppm}$ corresponding to $\mathrm{H}-4$, a signal that confirms the construction of the DHPM skeleton. As expected, the same proton absorbs at higher field ( $3.5 \mathrm{ppm})$ when aliphatic substituents are placed at C-4 position $\mathbf{8 h}$,i. Some other signals, especially $\mathrm{N}-\mathrm{H}$ protons, experience clearly marked shifts when running the NMR spectra in different solvents. In particular, $\mathrm{N}(3)-\mathrm{H}$ moves from $\sim 5.5 \mathrm{ppm}$ when the $\mathrm{NMR}$ is taken in $\mathrm{CDCl}_{3}$, to $\sim 9$ ppm when DMSO- $d_{6}$ was employed in order to improve the solubility of some of these derivatives. In view of the synthetic results summarized in Table 1, it can be firstly concluded that the reaction is clearly affected by two structural features: the substitution pattern of the urea component 6 and the nature of the aldehyde 7. Thus, when unsubstituted and monosubstituted ureas 6a,b were employed, the corresponding DHPM 8a-c,e-g,j-l was obtained satisfactorily (6393\%) with non-activated, activated and heteroaromatic aldehydes, but disappointingly when starting from deactivated aldehydes (DHPM 8d). It is known ${ }^{5 a}$ that simple monosubstituted alkyl ureas generally react well, and in a regioselective manner, to provide good yield of N(1)substitutedDHPMs. Contrarily, and as previously reported, the use of N,N'-disubstituted ureas 6c,d afforded heterocycles $\mathbf{8 m - t}$ in a diminished yield in all cases (21-51\%) regardless of the electronic nature of the aldehyde component 7 or the substitution of the 3 -ketoamide $5{ }^{18}$ The method under study seems to show also limitations with respect to the aliphatic nature of the aldehyde component. In both cases (DHPMs 8h,i), either the iso-propyl or the tert-butyl substituted analogs were prepared in only moderate yields. Finally, it should be emphasized that all assays carried out with unsubstituted ketoamide $\mathbf{5 b}$ pleasingly resulted once again in the formation of DHPMs $\mathbf{8 j}, \mathbf{k}$ in excellent yields.

\section{Conclusions}

The direct preparation of a series of DHPM-5-carboxamides by the Biginelli multicomponent reaction starting from acetylacetamides has been optimized with respect to the use of an adequate catalyst. This study has shown that the use of catalytic amounts of chloroacetic acid promotes the cyclocondensation reaction under solvent free conditions in most cases in good to excellent yields and with a superior behavior with respect to previously reported methods. The present direct approach avoids the hydrolysis/amidation steps that would be required to transform the most commonly employed 5-ethoxycarbonylDHPM derivatives into the final 5-carboxamidosubstituted dihydropyrimidines. 


\section{Experimental Section}

General. All reagents were purchased and used as received. Melting points were measured using open glass capillaries. Infrared spectra were recorded as $\mathrm{KBr}$ as thin films and peaks are reported

in $\mathrm{cm}^{-1}$. Only representative absorptions are given. NMR spectra were taken in DMSO- $d_{6}$ on a Bruker AC-300 instrument at $20-25^{\circ} \mathrm{C}$. Chemical shifts $(\delta)$ were measured in ppm relative to solvent $\left(\delta=2.50\right.$ for ${ }^{1} \mathrm{H}$ or 39.4 for ${ }^{13} \mathrm{C}$ ) as internal standard. Coupling constants, $J$, are reported in hertz. DEPT experiments were used to assist with the assignation of the signals and structural determinations.

\section{Typical procedure for the synthesis of DHPMs (8a-t)}

A mixture of 3-ketoamide $5(1 \mathrm{mmol})$, urea $6(1.5 \mathrm{mmol})$, aldehyde $7(1 \mathrm{mmol})$ and chloroacetic acid $(10 \mathrm{~mol} \%)$ was heated in an oil bath $\left(90^{\circ} \mathrm{C}\right)$ for $7-10 \mathrm{~h}$ in the absence of solvents. The progress of the reaction was monitored by TLC. When the reaction was completed, the flask was removed from the oil bath and allowed to stand at room temperature. Then, the mixture was poured into water and extracted with $\mathrm{CH}_{2} \mathrm{Cl}_{2}$. The organic extracts were dried with $\mathrm{Na}_{2} \mathrm{SO}_{4}$ (anh), solvent was evaporated under reduced pressure, and the resultant residue was purified, if necessary, by column chromatography (Hexanes/EtOAc, 3/7) followed by crystallization from the appropriate solvent.

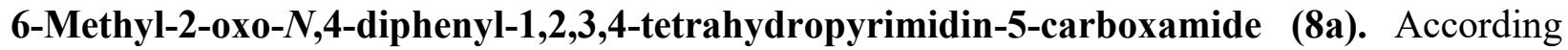
to the general procedure DHPM 8a was obtained from acetoacetanilide 5a, benzaldehyde $7 \mathbf{a}$ and urea 6a in $92 \%$ yield as an orange pale solid: $\mathrm{mp} 236-240{ }^{\circ} \mathrm{C}$ (hexanes, lit. ${ }^{13 \mathrm{c}} 239-240$ ).

\section{4-(3-Methoxyphenyl)-6-methyl-2-oxo- $N$-phenyl-1,2,3,4-tetrahydropyrimidin-5-}

carboxamide (8b). According to the general procedure DHPM 8b was obtained from acetoacetanilide 5a, 3-methoxybenzaldehyde $7 \mathbf{b}$ and urea $\mathbf{6 a}$ in $63 \%$ yield. This material had to be purified by column chromatography $\left(\mathrm{CH}_{2} \mathrm{Cl}_{2} / \mathrm{MeOH}, 9 / 1\right)$ and triturated with hexanes to afford DHPM 8b as a yellowish pure solid: $\mathrm{mp} 180-185^{\circ} \mathrm{C}$ (hexanes); IR (film) $v 3237,1672$, 1400, 1200; ${ }^{1} \mathrm{H}$ NMR (300 MHz, DMSO- $\left.d_{6}\right) 2.02$ (s, 3H), 3.67 (s, 3H), 5.37 (s, 1H), 6.52-6.80 (m, 4H), 6.82-6.99 (m, 3H), 7.20-7.23 (m, 3H), 8.33 (br s, 1H), $9.54(\mathrm{br} \mathrm{s}, 1 \mathrm{H}) ;{ }^{13} \mathrm{C}$ NMR $(75$ MHz, DMSO-d $d_{6}$ 17.5, 55.4, 105.8, 112.7, 112.7, 118.8, 120.0, 123.5, 129.0, 130.0, 138.9, 139.7, 149.3, 153.1, 159.8, 165.8; HRMS calcd for $\mathrm{C}_{19} \mathrm{H}_{19} \mathrm{~N}_{3} \mathrm{O}_{3} \cdot \mathrm{H}^{+} 338.1517$, found 338.1505.

6-Methyl-2-oxo- $N$-phenyl-4-(2-thienyl)-1,2,3,4-tetrahydropyrimidin-5-carboxamide (8c). According to the general procedure DHPM 8c was obtained from acetoacetanilide 5a, 2thiophenecarboxaldehyde $7 \mathbf{c}$ and urea $\mathbf{6 a}$ in $89 \%$ yield as a yellowish solid: mp $174-178{ }^{\circ} \mathrm{C}$ (hexanes); IR (film) v 3249, 1655, 1596; ${ }^{1} \mathrm{H}$ NMR (300 MHz, DMSO-d $) 2.04$ (s, 3H), 5.70 (s, $1 \mathrm{H}), 6.91-7.02(\mathrm{~m}, 3 \mathrm{H}), 7.22-7.37(\mathrm{~m}, 3 \mathrm{H}), 7.55-7.57(\mathrm{~m}, 2 \mathrm{H}), 7.79$ (br s, $1 \mathrm{H}), 8.85$ (br s, $1 \mathrm{H})$, 9.54 (br s, $1 \mathrm{H}$ ); ${ }^{13} \mathrm{C}$ NMR (75 MHz, DMSO-d $)$ 17.6, 51.0, 106.8, 120.2, 127.2, 129.0, 123.6, 124.0, 125.4, 139.7, 140.0, 149.2, 153.0, 165.4; HRMS calcd for $\mathrm{C}_{16} \mathrm{H}_{15} \mathrm{~N}_{3} \mathrm{O}_{2} \mathrm{~S} \cdot \mathrm{H}^{+} 314.0997$, found 314.0963 . 


\section{6-Methyl-4-(4-nitrophenyl)-2-oxo- $N$-phenyl-1,2,3,4-tetrahydropyrimidin-5-carboxamide}

(8d). According to the general procedure DHPM 8d was obtained from acetoacetanilide 5a, 4nitrobenzaldehyde 7d and urea 6a in 36\% yield as a yellowish solid: mp 307-309 ${ }^{\circ} \mathrm{C}$ (hexanes, lit. ${ }^{19}$ 304-306); IR (film) v 3364, 1720, 1518, 1533; ${ }^{1} \mathrm{H}$ NMR (300 MHz, DMSO-d6) 2.04 (s, $3 \mathrm{H}), 5.48(\mathrm{~s}, 1 \mathrm{H}), 6.96-7.01(\mathrm{~m}, 1 \mathrm{H}), 7.20-7.25(\mathrm{~m}, 2 \mathrm{H}), 7.50-7.53(\mathrm{~m}, 3 \mathrm{H}), 7.73$ (br s, 1H), 8.22 (d, $J=8.7,2 \mathrm{H}), 8.85$ (br s, 1H), 9.60 (br s, $1 \mathrm{H}) ;{ }^{13} \mathrm{C}$ NMR (75 MHz, DMSO-d 6 ) 17.6, 55.2, 104.9, $120.1,123.7,124.3,128.0,129.0,139.5,139.8,147.2,151.9,152.8,165.5$.

1,6-Dimethyl-2-oxo- $N$,4-diphenyl-1,2,3,4-tetrahydropyrimidin-5-carboxamide

(8e). According to the general procedure DHPM 8e was obtained from acetoacetanilide 5a, benzaldehyde 7a and N-methylurea $\mathbf{6 b}$ in $75 \%$ yield as a white solid: mp $216-222{ }^{\circ} \mathrm{C}$ (hexanes); IR (film) v 3295, 1696, 1626, 1434; ${ }^{1} \mathrm{H}$ NMR (300 MHz, DMSO-d $) 2.15$ (s, 3H), 3.05 (s, 3H), $5.28(\mathrm{~s}, 1 \mathrm{H}), 6.98-7.03(\mathrm{~m}, 1 \mathrm{H}), 7.16-7.35(\mathrm{~m}, 7 \mathrm{H}), 7.52-7.56(\mathrm{~m}, 2 \mathrm{H}), 7.71$ (br s, 1H), 9.80 (br s, $1 \mathrm{H}) ;{ }^{13} \mathrm{C}$ NMR (75 MHz, DMSO-d6) 17.1, 29.8, 54.4, 109.9, 120.1, 123.7, 126.5, 127.8, 129.0, 139.5, 139.6, 144.1, 154.0, 166.3; HRMS calcd for $\mathrm{C}_{19} \mathrm{H}_{19} \mathrm{~N}_{3} \mathrm{O}_{2} \cdot \mathrm{H}^{+} 322.1556$, found 322.1558.

4-(3-Hydroxyphenyl)-1,6-dimethyl-2-oxo- $N$-phenyl-1,2,3,4-tetrahydropyrimidin-5-

carboxamide (8f). According to the general procedure DHPM 8f was obtained from acetoacetanilide 5a, 3-hydroxybenzaldehyde 7e and N-methylurea $\mathbf{6 b}$ in $93 \%$ yield as a white solid: mp 238-242 ${ }^{\circ} \mathrm{C}$ (hexanes); IR (film) v 3301, 1676, 1626, 1434; ${ }^{1} \mathrm{H}$ NMR (300 MHz, DMSO-d6) 2.12 (s, 3H), $3.06(\mathrm{~s}, 3 \mathrm{H}), 5.20(\mathrm{~s}, 1 \mathrm{H}), 6.55-6.67(\mathrm{~m}, 3 \mathrm{H}), 6.96-7.08(\mathrm{~m}, 2 \mathrm{H}), 7.21-$ $7.28(\mathrm{~m}, 2 \mathrm{H}), 7.55-7.59(\mathrm{~m}, 2 \mathrm{H}), 7.65(\mathrm{br} \mathrm{s}, 1 \mathrm{H}), 9.37(\mathrm{~s}, 1 \mathrm{H}), 9.78(\mathrm{~s}, 1 \mathrm{H}) ;{ }^{13} \mathrm{C} \mathrm{NMR}(75 \mathrm{MHz}$, DMSO-d $\left.d_{6}\right) 17.1,29.8,54.3,110.1,113.3,114.77,116.9,120.1,123.7,129.0,129.9,139.3$, $139.7,145.7,154.1,158.0,166.4$.

\section{4-(2-Bromophenyl)-1,6-dimethyl-2-oxo- $N$-phenyl-1,2,3,4-tetrahydropyrimidin-5-}

carboxamide (8g). According to the general procedure DHPM 8g was obtained from acetoacetanilide 5a, 2-bromobenzaldehyde 7f and $\mathrm{N}$-methylurea $\mathbf{6 b}$ in $75 \%$ yield as a white solid: mp 201-204 ${ }^{\circ} \mathrm{C}$ (hexanes); IR (film) v 3211, 1693, 1627; ${ }^{1} \mathrm{H}$ NMR (300 MHz, DMSO-d $)$ $2.13(\mathrm{~s}, 3 \mathrm{H}), 3.10(\mathrm{~s}, 3 \mathrm{H}), 5.64(\mathrm{~s}, 1 \mathrm{H}), 6.95-7.02(\mathrm{~m}, 1 \mathrm{H}), 7.15-7.54(\mathrm{~m}, 8 \mathrm{H}), 7.61(\mathrm{~s}, 1 \mathrm{H}), 9.89$ $(\mathrm{s}, 1 \mathrm{H}) ;{ }^{13} \mathrm{C}$ NMR $\left(75 \mathrm{MHz}, \mathrm{DMSO}-d_{6}\right)$ 17.1, 29.8, 54.6, 109.0, 120.0, 120.1, 122.2, 123.7, $128.8, \quad 129.0,130.1,133.1,139.3,139.5,142.7,153.4,165.8 ;$ HRMS calcd for $\mathrm{C}_{19} \mathrm{H}_{18}{ }^{79} \mathrm{BrN}_{3} \mathrm{O}_{2} \cdot \mathrm{H}^{+} 400.0668$, found 400.0661 .

1,6-Dimethyl-4-isopropyl-2-oxo- $N$-phenyl-1,2,3,4-tetrahydropyrimidin-5-carboxamide (8h). According to the general procedure DHPM $\mathbf{8 h}$ was obtained from acetoacetanilide 5a, isobutyraldehyde $\mathbf{7 g}$ and N-methylurea $\mathbf{6 b}$ in $27 \%$ yield. This material had to be purified by column chromatography (EtOAc/hexanes, 3/7) and triturated in hexanes to afford DHPM $\mathbf{8 h}$ as a white pure solid: mp 239-241 ${ }^{\circ} \mathrm{C}$ (hexanes); IR (film) $v 3305,1692,1653 ;{ }^{1} \mathrm{H} \mathrm{NMR} \mathrm{(300} \mathrm{MHz,}$ DMSO-d6) 0.77-0.83 (m, 6H), 1.52-1.62 (m, 1H), 2.07 (s, 3H), 2.93 (s, 3H), $3.96(\mathrm{~s}, 1 \mathrm{H}), 7.02-$ $7.04(\mathrm{~m}, 1 \mathrm{H}), 7.20-7.32(\mathrm{~m}, 3 \mathrm{H}), 7.57-7.64(\mathrm{~m}, 2 \mathrm{H}), 9.78(\mathrm{~s}, 1 \mathrm{H}) ;{ }^{13} \mathrm{C}$ NMR $(75 \mathrm{MHz}, \mathrm{DMSO}-$ $\left.d_{6}\right) 16.9,17.0,18.2,29.7,35.0,56.1,109.3,120.9,123.7,129.0,139.5,139.8,154.7,167.3$; HRMS calcd for $\mathrm{C}_{16} \mathrm{H}_{21} \mathrm{~N}_{3} \mathrm{O}_{2} \cdot \mathrm{H}^{+} 288.1723$, found 288.1712 . 
4-tert-Butyl-1,6-dimethyl-2-oxo- $N$-phenyl-1,2,3,4-tetrahydropyrimidin-5-carboxamide (8i). According to the general procedure DHPM $\mathbf{8 h}$ was obtained from acetoacetanilide $\mathbf{5 a}$, pivalaldehyde $7 \mathbf{h}$ and N-methylurea $\mathbf{6 b}$ in $47 \%$ yield. This material had to be purified by column chromatography (EtOAc/hexanes, 9/1) and triturated in hexanes to afford DHPM 8i as a white pure solid: mp 259-262 ${ }^{\circ} \mathrm{C}$ (hexanes); IR (film) v 3290, 1675, 1653; ${ }^{1} \mathrm{H}$ NMR (300 MHz, DMSO-d6) $0.80(\mathrm{~s}, 9 \mathrm{H}), 2.09(\mathrm{~s}, 3 \mathrm{H}), 2.96(\mathrm{~s}, 3 \mathrm{H}), 3.83(\mathrm{~s}, 1 \mathrm{H}), 7.01-7.04(\mathrm{~m}, 1 \mathrm{H}), 7.24-7.30(\mathrm{~m}$, 3H), 7.57-7.60 (m, 2H), 9.78 (s, 1H); ${ }^{13} \mathrm{C}$ NMR (75 MHz, DMSO-d6) 16.8, 25.4, 29.7, 38.7, 59.5, 109.3, 120.1, 123.6, 129.0, 139.8, 140.2, 155.1, 168.1; HRMS calcd for $\mathrm{C}_{17} \mathrm{H}_{23} \mathrm{~N}_{3} \mathrm{O}_{2} \cdot \mathrm{H}^{+}$ 302.1873 , found 302.1869 .

6-Methyl-2-oxo-4-phenyl-1,2,3,4-tetrahydropyrimidin-5-carboxamide $(8 \mathbf{j})$. According to the general procedure DHPM 8j was obtained from acetoacetamide $\mathbf{5 b}$, benzaldehyde $7 \mathbf{a}$ and urea $\mathbf{6 a}$ in $94 \%$ yield as a white solid: mp 218-224 ${ }^{\circ} \mathrm{C}$ (hexanes); ${ }^{1} \mathrm{H}$ NMR (300 MHz, DMSO- $\left.d_{6}\right) 2.04$ (s, $3 \mathrm{H}), 5.21(\mathrm{~s}, 1 \mathrm{H}), 6.82$ (br s, 2H), 7.20-7.34 (m, 5H), 7.42-7.51 (s, 1H), $8.54(\mathrm{~s}, 1 \mathrm{H})$; ${ }^{13} \mathrm{C} \mathrm{NMR}$ (75 MHz, DMSO- $d_{6}$ ) 17.6, 55.1, 104.9, 126.9, 127.6, 128.8, 139.3, 144.9, 153.2, 168.6; HRMS calcd for $\mathrm{C}_{12} \mathrm{H}_{13} \mathrm{~N}_{3} \mathrm{O}_{2} \cdot \mathrm{H}^{+} 232.1086$, found 232.1093.

1,6-Dimethyl-2-oxo-4-phenyl-1,2,3,4-tetrahydropyrimidin-5-carboxamide (8k). According to the general procedure DHPM $\mathbf{8 k}$ was obtained from acetoacetamide $\mathbf{5 b}$, benzaldehyde $\mathbf{7 a}$ and Nmethylurea $6 \mathbf{b}$ in $73 \%$ yield as a white solid: $\mathrm{mp} 252-259{ }^{\circ} \mathrm{C}$ (hexanes); IR (film) $v 3375,1690$, 1654, 1396; ${ }^{1} \mathrm{H}$ NMR (300 MHz, DMSO-d $)$ ): 2.18 (s, 3H), 3.01 (s, 3H) 5.15 (s, 1H), 6.96 (br s, $2 \mathrm{H}), 7.20-7.32(\mathrm{~m}, 5 \mathrm{H}), 7.64(\mathrm{~s}, 1 \mathrm{H}) ;{ }^{13} \mathrm{C} \mathrm{NMR}$ (75 MHz, DMSO- $\left.d_{6}\right)$ 16.8, 29.8, 54.0, 109.2, 126.6, 127.7, 128.8, 139.9, 144.2, 154.1, 169.2; HRMS calcd for $\mathrm{C}_{13} \mathrm{H}_{15} \mathrm{~N}_{3} \mathrm{O}_{2} \cdot \mathrm{H}^{+} 246.1243$, found 246.1240 .

4-(3-Methoxyphenyl)-1,6-dimethyl-2-oxo- $N$-phenyl-1,2,3,4-tetrahydropyrimidin-5carboxamide (81). According to the typical procedure DHPM 81 was obtained from acetoacetanilide 5a, 3-methoxybenzaldehyde $\mathbf{7 b}$ and $\mathrm{N}$-methylurea $\mathbf{6 b}$ in $97 \%$ yield as a pale brown solid after triturating of the residue from hexanes: mp 148-152 ${ }^{\circ} \mathrm{C}$ (hexanes); IR (film) $v$ 3290, 1655, 1488; ${ }^{1} \mathrm{H}$ NMR (300 MHz, $\left.\mathrm{CDCl}_{3}\right) 2.29$ (s, 3H), 3.23 (s, 3H), 3.77 (s, 3H), 5.30 (s, $1 \mathrm{H}), 6.55-7.25(\mathrm{~m}, 11 \mathrm{H}) ;{ }^{13} \mathrm{C} \mathrm{NMR}\left(75 \mathrm{MHz}, \mathrm{CDCl}_{3}\right)$ 16.7, 29.9, 55.2, 55.6, 109.0, 112.1, 113.8, $118.4,119.9,124.4,128.9,130.4,137.7,140.2,143.9,153.8,160.3,165.7 ; \mathrm{MS}[\mathrm{M}+1] \mathrm{m} / z: 352$ (100), 309 (32), 259 (79), 233 (31), 122 (35), 94 (56); HRMS calcd for $\mathrm{C}_{20} \mathrm{H}_{21} \mathrm{~N}_{3} \mathrm{O}_{3} \cdot \mathrm{H}^{+}$: 352.1661, found: 352.1670 .

4-(3-Methoxyphenyl)-1,3,6-trimethyl-2-oxo- $N$-phenyl-1,2,3,4-tetrahydropyrimidin-5carboxamide (8m). According to the typical procedure DHPM 8m was obtained from acetoacetanilide 5a, 3-methoxybenzaldehyde $\mathbf{7 b}$ and N,N'-dimethylurea $\mathbf{5 c}$ in 36\% yield as a pale brown solid after purification by column chromatography (Hexanes/EtOAc, 3/7) followed by crystallization from hexanes: $\mathrm{mp} 66-68{ }^{\circ} \mathrm{C}$ (hexanes); IR (film) $v 3290,1648,1532 ;{ }^{1} \mathrm{H}$ NMR $\left(300 \mathrm{MHz}, \mathrm{CDCl}_{3}\right) 2.22$ (s, 3H), 2.88 (s, 3H), 3.21 (s, 3H), 3.71 (s, 3H), 5.08 (s, 1H), 6.78-6.80 $(\mathrm{m}, 3 \mathrm{H}), 7.00-7.09(\mathrm{~m}, 2 \mathrm{H}), 7.21-7.33(\mathrm{~m}, 5 \mathrm{H}) ;{ }^{13} \mathrm{C} \mathrm{NMR}(75 \mathrm{MHz}, \mathrm{CDCl} 3)$ 16.6, 30.1, 33.9, 55.2, 62.3, 108.7, 112.4, 113.3, 118.7, 119.8, 124.4, 129.9, 130.4, 137.7, 140.0, 141.8, 153.7, 
160.3, 165.7; MS [M+1] m/z: 366 (64), 350 (30), 309 (79), 233 (30), 273 (100); HRMS calcd for $\mathrm{C}_{21} \mathrm{H}_{23} \mathrm{~N}_{3} \mathrm{O}_{3} \cdot \mathrm{H}^{+}: 366.1827$, found: 366.1818 .

\section{1,3,6-Trimethyl-2-oxo- $N$,4-diphenyl-1,2,3,4-tetrahydropyrimidin-5-carboxamide}

(8n). According to the typical procedure DHPM 8n was obtained from acetoacetanilide 5a, benzaldehyde 7a and N,N'-dimethylurea $\mathbf{6 c}$ in $43 \%$ yield as a yellowish oil after purification by column chromatography (Hexanes/EtOAc, 3/7): IR (film) v 3295, 1654, 1540; ${ }^{1} \mathrm{H}$ NMR (300 $\left.\mathrm{MHz}, \mathrm{CDCl}_{3}\right) 2.22(\mathrm{~s}, 3 \mathrm{H}), 2.88(\mathrm{~s}, 3 \mathrm{H}), 3.21(\mathrm{~s}, 3 \mathrm{H}), 5.10(\mathrm{~s}, 1 \mathrm{H}), 6.80-6.91$ (br s, 1H), 7.00$7.41(\mathrm{~m}, 10 \mathrm{H}) ;{ }^{13} \mathrm{C} \mathrm{NMR}\left(75 \mathrm{MHz}, \mathrm{CDCl}_{3}\right)$ 16.7, 30.6, 34.2, 62.4, 108.9, 119.9, 124.4, 124.6, 126.5, 128.7, 129.0, 129.5, 137.7, 140.0, 140.2, 153.7, 165.7; MS [M+1] m/z: 336 (73), 335 (33), 320 (32), 243 (100); HRMS calcd for $\mathrm{C}_{20} \mathrm{H}_{21} \mathrm{~N}_{3} \mathrm{O}_{2} \cdot \mathrm{H}^{+}: 336.1712$, found: 336.1707.

\section{$\mathrm{N}$-(p-Nitrophenyl)-1,3,6-trimethyl-2-oxo-4-phenyl-1,2,3,4-tetrahydropyrimidin-5-}

carboxamide (8o). According to the typical procedure DHPM 80 was obtained from N-pnitrophenylacetoacetamide 5c, benzaldehyde 7a and N,N'-dimethylurea $\mathbf{6 c}$ in $42 \%$ yield as a yellowish solid after purification by column chromatography (Hexanes/EtOAc, 3/7) followed by crystallization from hexanes: $\mathrm{mp} 224-228{ }^{\circ} \mathrm{C}$ (hexanes); IR (film) $v 3266,1646,1501$; ${ }^{1} \mathrm{H}$ NMR $\left(300 \mathrm{MHz} \mathrm{CDCl}_{3}\right) 2.31(\mathrm{~s}, 3 \mathrm{H}), 2.88(\mathrm{~s}, 3 \mathrm{H}), 3.28(\mathrm{~s}, 3 \mathrm{H}), 5.07(\mathrm{~s}, 1 \mathrm{H}), 7.24-7.31(\mathrm{~m}, 3 \mathrm{H})$, 7.35$7.41(\mathrm{~m}, 2 \mathrm{H}), 7.46-7.53(\mathrm{~m}, 2 \mathrm{H}), 7.58-7.62$ (br s, $1 \mathrm{H}), 8.10-8.16(\mathrm{~m}, 2 \mathrm{H}) ;{ }^{13} \mathrm{C} \mathrm{NMR}(75 \mathrm{MHz}$, $\left.\mathrm{CDCl}_{3}\right)$ 30.7, 34.8, 60.5, 64.6, 98.9, 126.4, 128.6, 128.7, 129.0, 130.9, 137.4, 137.8, 138.9, 151.9, 158.1, 169.6; (Compound 6e resulted to be unstable under all conditions employed to register its MS/HR mass spectrum).

\section{1,3-Diethyl-4-(3-methoxyphenyl)-6-methyl-2-oxo- $N$-phenyl-1,2,3,4-tetrahydropyrimidin-5-} carboxamide (8p). According to the typical procedure DHPM 8p was obtained from acetoacetanilide 5a, 3-methoxybenzaldehyde $7 \mathbf{b}$ and N,N'-diethylurea $\mathbf{6 d}$ in $21 \%$ yield as a pale brown solid after purification by column chromatography (Hexanes/EtOAc, 3/7) followed by crystallization from hexanes: $\mathrm{mp} 64-70{ }^{\circ} \mathrm{C}$ (hexanes); IR (film) $v$ 2916, 1668, 1596; ${ }^{1} \mathrm{H}$ NMR $\left(300 \mathrm{MHz}, \mathrm{CDCl}_{3}\right)$ 1.00-1.14 (m, 3H), 1.19-1.26 (m, 3H), 2.23-2.37 (m, 1H), 2.88-2.94 (m, 1H), 3.63-3.70 (m, 1H), $3.74(\mathrm{~s}, 3 \mathrm{H}), 3.94(\mathrm{~m}, 1 \mathrm{H}), 5.12(\mathrm{~s}, 1 \mathrm{H}), 6.83-6.92(\mathrm{~m}, 4 \mathrm{H}), 7.02-7.14(\mathrm{~m}$, 1H), 7.22-7.32 (m, 5H); ${ }^{13} \mathrm{C}$ NMR (75 MHz, $\left.\mathrm{CDCl}_{3}\right)$ 12.7, 15.2, 16.2, 38.0, 41.3, 55.3, 59.9, 100.0, 109.1, 112.2, 114.0, 119.0, 124.4, 129.1, 130.3, 137.6, 139.3, 143.3, 152.6, 160.4, 166.0; MS [M+1] m/z: 394 (100), 393 (31), 378 (33), 323 (40), 301 (71); HRMS calcd for $\mathrm{C}_{23} \mathrm{H}_{27} \mathrm{~N}_{3} \mathrm{O}_{3} \cdot \mathrm{H}^{+}$: 394.2130, found: 394.2115.

\section{$\mathrm{N}$-(2,4-dimethylphenyl)-4-phenyl-1,3,6-trimethyl-2-oxo-1,2,3,4-tetrahydropyrimidin-5-}

carboxamide (8q). According to the typical procedure DHPM 8q was obtained from N-(2,4dimethylphenyl)acetoacetamide 5d, 3-methoxybenzaldehyde $\mathbf{7 b}$ and N,N'-dimethylurea $\mathbf{6 c}$ in $51 \%$ yield as a yellowish solid after purification by column chromatography (Hexanes/EtOAc, 3/7) followed by crystallization from hexanes: $\mathrm{mp} 185-190{ }^{\circ} \mathrm{C}$ (hexanes); IR (film) $v 3261$, 1655, 1515; ${ }^{1} \mathrm{H}$ NMR (300 MHz, $\left.\mathrm{CDCl}_{3}\right) 1.78$ (s, 3H), 2.31 (s, 3H), 2.34 (s, 3H), 2.87 (s, 3H), $3.23(\mathrm{~s}, 3 \mathrm{H}), 5.07(\mathrm{~s}, 1 \mathrm{H}), 6.49-6.56(\mathrm{~m}, 1 \mathrm{H}), 6.83-7.00(\mathrm{~m}, 2 \mathrm{H}), 7.20-7.41(\mathrm{~m}, 5 \mathrm{H}), 7.46-7.53$ $(\mathrm{m}, 1 \mathrm{H}) ;{ }^{13} \mathrm{C} \mathrm{NMR}\left(75 \mathrm{MHz}, \mathrm{CDCl}_{3}\right)$ 16.7, 17.3, 20.8, 30.5, 34.1, 62.4, 108.6, 123.3, 126.7, 
$127.1,128.5,129.3,129.5,131.0,133.3,135.0,139.7,139.9,153.7,166.0 ; \mathrm{MS}[\mathrm{M}+1] \mathrm{m} / z: 364$ (100), 363 (42), 243 (61); HRMS calcd for $\mathrm{C}_{22} \mathrm{H}_{25} \mathrm{~N}_{3} \mathrm{O}_{2} \cdot \mathrm{H}^{+}: 364.2025$, found: 364.2027.

1,3,6-Trimethyl-2-oxo-4-phenyl-1,2,3,4-tetrahydropyrimidin-5-carboxamide (8r). According to the typical procedure DHPM 8r was obtained from acetoacetamide $\mathbf{5 b}$, benzaldehyde $\mathbf{7 a}$ and N,N'-dimethylurea $\mathbf{6 c}$ in $23 \%$ yield as a yellowish solid after purification by column chromatography (Hexanes/EtOAc, 3/7) followed by crystallization from hexanes: mp 204-208 ${ }^{\circ} \mathrm{C}$ (hexanes); IR (film) v 3197, 1646, 1601; $\left.{ }^{1} \mathrm{H} \mathrm{NMR} \mathrm{(300} \mathrm{MHz,} \mathrm{CDCl}_{3}\right) 2.29$ (s, 3H), 2.86 (s, 3H), 3.20 (s, 3H), 5.04 (s, 1H), 5.36-5.57 (br s, 2H), 7.20-7.32 (m, 5H); ${ }^{13} \mathrm{C} \mathrm{NMR}\left(75 \mathrm{MHz}, \mathrm{CDCl}_{3}\right)$ $16.7,30.7,34.2,62.7,107.3,126.6,128.5,129.2,139.9,141.4,153.7,169.4 ; \mathrm{MS}[\mathrm{M}+1] \mathrm{m} / z: 260$ (91), 244 (100), 242 (96), 378 (33), 203 (64), 182 (59), 164 (55); HRMS calcd for $\mathrm{C}_{14} \mathrm{H}_{17} \mathrm{~N}_{3} \mathrm{O}_{2} \cdot \mathrm{H}^{+}: 260.1399$, found: 260.1406 .

\section{1,3,6-Trimethyl-4-(2-thienyl)-2-oxo- $N$-phenyl-1,2,3,4-tetrahydropyrimidin-5-carboxamide}

(8s). According to the typical procedure DHPM 8s was obtained from acetoacetanilide 5a, 2thienylcarboxaldehyde 7c and N,N'-dimethylurea $\mathbf{6 b}$ in $40 \%$ yield as a pale brown solid after purification by column chromatography (Hexanes/EtOAc, 1/1) followed by crystallization from hexanes: mp 137-140 ${ }^{\circ} \mathrm{C}$ (hexanes); IR (film) v 3285, 1653, 1438; ${ }^{1} \mathrm{H}$ NMR $\left(300 \mathrm{MHz}, \mathrm{CDCl}_{3}\right)$ 2.35 (s, 3H), 2.97 (s, 3H), $3.25(\mathrm{~s}, 3 \mathrm{H}), 5.33(\mathrm{~s}, 1 \mathrm{H}), 6.97-7.10(\mathrm{~m}, 3 \mathrm{H}), 7.26-7.36(\mathrm{~m}, 6 \mathrm{H}) ;{ }^{13} \mathrm{C}$ NMR (75 MHz, $\left.\mathrm{CDCl}_{3}\right)$ 16.4, 30.6, 34.2, 57.4, 108.2, 119.8, 124.3, 125.9, 126.5, 127.1, 129.0, 137.7, 142.4, 143.1, 153.7, 169.4; MS [M+1] m/z: 342 (100), 341 (45), 285 (34), 258 (65), 249 (100); HRMS calcd for $\mathrm{C}_{18} \mathrm{H}_{19} \mathrm{~N}_{3} \mathrm{O}_{2} \mathrm{~S} \cdot \mathrm{H}^{+}: 342.1276$, found: 342.1282 .

\section{1,3,6-Trimethyl-4-(o-nitrophenyl)-2-oxo- $N$-phenyl-1,2,3,4-tetrahydropyrimidin-5-}

carboxamide (8t). According to the typical procedure DHPM 8t was obtained from acetoacetanilide 5a, 2-nitrobenzaldehyde 7i and N,N'-dimethylurea $\mathbf{6 c}$ in $42 \%$ yield as a pale brown solid after purification by column chromatography (Hexanes/EtOAc, 2/8) followed by crystallization from hexanes: $\mathrm{mp} 236-239{ }^{\circ} \mathrm{C}$ (hexanes); IR (film) $v 3285,1653,1437 ;{ }^{1} \mathrm{H} \mathrm{NMR}$ $\left(300 \mathrm{MHz}, \mathrm{CDCl}_{3}\right) 2.40(\mathrm{~s}, 3 \mathrm{H}), 2.84(\mathrm{~s}, 3 \mathrm{H}), 3.29$ (s, 3H), $6.04(\mathrm{~s}, 1 \mathrm{H}), 7.03-7.12(\mathrm{~m}, 1 \mathrm{H}), 7.23-$ $7.30(\mathrm{~m}, 3 \mathrm{H}), 7.37-7.68(\mathrm{~m}, 5 \mathrm{H}), 7.74-7.84(\mathrm{~m}, 1 \mathrm{H}) ;{ }^{13} \mathrm{C} \mathrm{NMR}\left(75 \mathrm{MHz}, \mathrm{CDCl}_{3}\right)$ 17.1, 30.8, 33.9, 56.3, 106.3, 120.2, 124.3, 124.5, 128.9, 129.5, 129.6, 134.1, 134.6, 137.6, 143.1, 153.0, 164.0, 176.2; MS [M+1] m/z: 381 (100), 363 (36), 349 (33), 228 (40), 301 (65); HRMS calcd for $\mathrm{C}_{20} \mathrm{H}_{20} \mathrm{~N}_{4} \mathrm{O}_{4} \cdot \mathrm{H}^{+}: 381.1563$, found: 381.1565 .

\section{Supporting Information}

Copies of NMR spectra for compounds 8a-t are included. 


\section{Acknowledgements}

Financial support from the Basque Government (GIU 06/87), and the Spanish Ministry of Science and Innovation CTQ2007-64501/BQU) is gratefully acknowledged. The authors gratefully acknowledge PETRONOR, S. A. (Muskiz, Bizkaia) for the generous gift of hexanes. I. C. thanks also the Spanish Ministry for a grant.

\section{References and Notes}

1. (a) Ganem, B. Acc. Chem. Res. 2009, 42, 463. (b) Armstrong, R. W.; Combs, A. P.; Tempest, P. A.; Brown, S. D.; Keating, T. A. Acc. Chem. Res. 1996, 29, 123.

2. Biginelli, P. Gazz. Chim. Ital. 1893, 23, 360.

3. Kappe, C. O. Eur. J. Med. Chem. 2000, 35, 1043.

4. (a) Atwal, K. S.; Rovnyak, G. C.; Kimball, S. D.; Floyd, D. M.; Moreland, S.; Swanson, B. N.; Gougoutas, J. Z.; Schwartz, J.; Smillie, K. M.; Malley, M. F. J. Med. Chem., 1990, 33, 2629. (b) Rovnyak, G. C.; Kimball, S. D.; Beyer, B.; Cucinotta, G.; DiMarco, J. D.; Gougoutas, J. Z.; Hedberg, A.; Malley, M. F.; McCarthy, J. P.; Zhang, R.; Moreland, S. J. Med. Chem. 1995, 38, 119, and references cited therein.

5. (a) Kappe, C. O. Org. React. 2004, 63, 1. (b) C. O. Kappe, Acc. Chem. Res. 2000, 33, 879. (c) Kappe, C. O. Tetrahedron 1993, 49, 6937.

6. See, for example: Overman, L. E.; Rabinowitz, M. H.; Renhowe, P. A. J. Am. Chem. Soc. 1995, 117, 2657.

7. The "Atwal modification" takes place between a preformed enone and a protected urea or thiourea. See, for example: (a) O'Reilly, B. C.; Atwal, K. S. Heterocycles 1987, 26, 1185. (b) Atwal, K. S.; O'Reilly, B. C.; Gougoutas, J. Z.; Malley, M. F. Heterocycles 1987, 26 , 1189. (c) For a stepwise strategy based on the Mannich reaction of 3-ketoesteres and acylimines, see: Goss, J. M.; Scahus, S. E. J. Org. Chem. 2008, 73, 7651.

8. The original conditions described by Biginelli required the use of $\mathrm{HCl}$ activation in refluxing ethanol, but more sophisticated recent protocols has been described. (a) For soluble polymer-supported synthesis, see: Wang, Y. G.; Xia, M. Tetrahedron Lett. 2002, 43, 7703. (b) For solvent-free microwave-irradiated conditions, see: Stadler, A.; Kappe, C. O. J. Comb. Chem. 2001, 3, 624. (c) The preparation of Biginelli compounds under sonochemical activation has been described in: Zhidovinova, M. S.; Fedorova, O. V.; Rusinov, G. L.; Ovchinnikova, I. G. Mol. Divers. 2003, 6, 323. (d) The Biginelli condensation takes place as well in ionic liquids. See, for example: Peng, J.; Deng, Y. Tetrahedron Lett. 2001, 42, 5917.

9. Many of the improved protocols involve the use of Lewis acids instead of the traditional mineral acid catalysts. For a recent review, see: Vdovina, S. V.; Mamedov, V. A. Russ. Chem. Rev. 2008, 77, 1017 and references cited therein. 
10. (a) Klein, E.; DeBonis, S.; Thiede, B.; Skoufias, D.; Kozielski, F.; Lebeau, L. Bioorg. Med. Chem. 2007, 15, 6474. (b) Desai, B.; Dallinger, D.; Kappe, C. O. Tetrahedron 2006, 62, 4651. (c) Desai, B.; Kappe, C. O. J. Comb. Chem. 2005, 7, 641.

11. Barrow, J. C.; Nantermet, P. G.; Selnick, H. G.; Glass, K. L.; Rittle, K. E.; Gilbert, K. F.; Steele, T. G.; Homnick, C. F.; Freidinger, R. M.; Ransom, R. W.; Kling, P.; Reiss, D.; Broten, T. P.; Schorn, T. W.; Chang, R. S. L.; O’Malley, S. S.; Olah, T.; Ellis, J. D.; Barrish, A.; Kassahun, K.; Leppert, P.; Nagarathnam, D.; Forray, C. J. Med. Chem. 2000, 43, 2703.

12. Haggarty, S. J.; Mayer, T. U.; Miyamoto, D. T.; Fathi, R.; King, R. W.; Mitchison, T. J.; Schreiber, S. L. Chem. Biol. 2000, 7, 275.

13. (a) Pansuriya, A. M.; Savant, M. M.; Bhuva, C. V.; Singh, J.; Naliapara, Y. T. Arkivoc 2009, (vii), 70. (b) Schmidt, R. J.; Lombardo, L. J.; Traeger, S. C.; Williams, D. K. Tetrahedron Lett. 2008, 49, 3009. (c) Amini, M. M.; Shaabani, A.; Bazgir, A. Catal. Commun. 2006, 7 , 843. (d) Chevanov, V. A.; Muravyova, E. A.; Desenko, S. M.; Mustov, V. I.; Knyazeva, I. V.; Shishkina, S. V.; Shishkin, O. V.; Kappe, C. O. J. Comb. Chem. 2006, 8, 427.

14. Duburs, G.; Khanina, E. L. Khim. Geterotsikl. Soedin. 1976, 220; Chem. Abstr. 1976, 85, 32946.

15. Kappe, C. O.; Stadler, A. Methods in Enzymology 2003, 369 (Part B), 197.

16. Kozhevnikov, I. V. in Catalysis for Fine Chemical Synthesis; Catalysis by Polyoxometalates, Roberts, S. M.; Kozhevnikov, I. V. and Derouane, E. (Series Eds.), Wiley: New York, 2002; Vol. 2, pp 216.

17. (a) Tanaka, K.; Toda, F. Chem. Rev. 2000, 100, 1025. (b) Cave, G. W. V.; Raston, C. L.; Scott, J. L. Chem. Commun. 2001, 2159. (c) Metzger, J. O. In Organic Synthesis Highlights $V$; Schmalz, H. -G., Wirth, T., Eds.; Wiley-VCH: Weinheim, 2003; p 82. (d) Martins, M. A. P.; Frizzo, C. P.; Moreira, D. N.; Buriol, L.; Machado, P. Chem. Rev. 2009, 109, 4140.

18. Literature shows examples directed to circumvent this problem. Singh, K.; Arora, D.; Singh, S. Tetrahedron Lett. 2006, 47, 4205. In our hands, the application of the reported conditions was not successful at all.

19. Previously prepared in: Virsodia, V.; Pissurlenkar, R. R. S.; Manvar, D.; Dholakia, C.; Adlakha, P.; Shah, A.; Coutinho, E. C. Eur. J. Med. Chem. 2008, 43, 2103. 